Cahiers $d u$ MONDE RUSSE

\section{Cahiers du monde russe}

Russie - Empire russe - Union soviétique et États indépendants

$52 / 4 \mid 2011$

Varia

\title{
Maria Zalambani, Censura, istituzioni e politica letteraria in URSS (1964-1985)
}

\section{Emilia Koustova}

\section{OpenEdition}

\section{Journals}

Édition électronique

URL : http://journals.openedition.org/monderusse/7647

DOI : $10.4000 /$ monderusse. 7647

ISSN : $1777-5388$

\section{Éditeur}

Éditions de l'EHESS

\section{Édition imprimée}

Date de publication : 20 décembre 2011

Pagination : 795-797

ISBN : 978-2-7132-2353-2

ISSN : $1252-6576$

\section{Référence électronique}

Emilia Koustova, « Maria Zalambani, Censura, istituzioni e politica letteraria in URSS (1964-1985)», Cahiers du monde russe [En ligne], 52/4 | 2011, mis en ligne le 03 décembre 2012, Consulté le 25 septembre 2020. URL : http://journals.openedition.org/monderusse/7647 ; DOI : https://doi.org/ 10.4000/monderusse.7647

Ce document a été généré automatiquement le 25 septembre 2020. 


\title{
Maria Zalambani, Censura, istituzioni e politica letteraria in URSS (1964-1985)
}

\author{
Emilia Koustova
}

\section{RÉFÉRENCE}

Maria ZALAMBANI, Censura, istituzioni e politica letteraria in URSS (1964-1985).

Firenze : Firenze University Press, 2009, 284 p.

1 Dans son dernier ouvrage, Maria Zalambani, spécialiste de la littérature soviétique à l'université de Bologne, propose une synthèse portant sur la censure littéraire soviétique durant la période de la «stagnation» qui fait actuellement l'objet d'un certain renouvellement historiographique. L'approche choisie permet à l'auteur de mettre en évidence de nombreuses thématiques autour de la censure, au-delà de son histoire institutionnelle ou des politiques soviétiques ciblant le domaine littéraire. Les questions soulevées relèvent souvent de l'histoire sociale, les phénomènes observés dans les rapports entre les champs politique et culturel pouvant apporter de précieux éléments de compréhension des processus à l'œuvre dans la société soviétique qu'on a longtemps crue endormie.

2 Cette synthèse est réalisée à partir de nombreux matériaux publiés (archives, Mémoires...) et d'une littérature, en premier lieu russophone, consacrée à la censure et à la politique culturelle soviétique ${ }^{1}$. L'analyse et l'organisation de l'ouvrage sont largement inspirées de la pensée de Pierre Bourdieu. En raisonnant en termes de "champ du pouvoir» et de "champ de la production culturelle », l'auteur constate que, si dans le cas soviétique le dernier est particulièrement dominé et contrôlé par le premier, il n'en est pas moins en proie à une lutte entre principes hétéronomes et autonomes de hiérarchisation, entre forces centripètes et centrifuges. La censure apparaît alors comme l'expression par excellence de la tendance centripète, qui 
cherche à maintenir un contrôle extérieur, alors que les tendances autonomes et centrifuges, qui s'expriment avec une force particulière durant les années Brežnev, donnent lieu à la naissance de "nouvelles formes artistiques indépendantes " et à différents mouvements qui cherchent à ignorer, à contourner, sinon à défier la censure (p. 18, 52).

Ce choix théorique se révèle fructueux car il permet d'éviter une approche purement descriptive et chronologique de l'évolution des mécanismes de la censure, mais aussi d'adopter une vision complexe de celle-ci, en considérant que ses acteurs en sont des institutions variées, du KGB à la critique littéraire, en distinguant les différentes fonctions de la censure (répressive, prescriptive), et surtout en soulignant l'existence de l'autocensure pratiquée par chacun ("censore dell'anima»), qui exerce un contrôle sur la production culturelle souvent bien plus efficace que toute institution publique (p. 49).

4 La monographie s'ouvre donc par l'analyse de l'évolution des champs du pouvoir et de la production culturelle et une rapide évocation de la politique culturelle du parti durant les deux dernières décennies soviétiques. Cette partie introductive permet d'esquisser un cadre et d'évoquer des éléments de chronologie, utiles notamment aux non-spécialistes.

5 Vient ensuite l'examen des « institutions de la censure » : le Glavlit bien-sûr (principal organisme chargé de la censure de 1922 à 1991), mais aussi le KGB, l'Union des écrivains, les rédacteurs, les traducteurs et les critiques littéraires, sans oublier cette « anti-institution » que constitue le samizdat. Bien que l'analyse de leur fonctionnement soit souvent très (trop) rapide, ce choix présente l'avantage de rendre compte de la multiplicité et de la complexité des formes et des mécanismes de la censure soviétique, en rappelant les particularités et l'importance, de ce point de vue, de certaines institutions, comme celle des éditeurs (redaktory). En effet, dans la production littéraire soviétique, ceux-ci occupent une position très ambigüe, car ce sont eux qui réalisent l'essentiel de la censure lors du travail éditorial sur le manuscrit en vue de sa publication.

6 Le travail de censure est notamment conditionné par l'existence de nombreux sujets-tabous, étudiés rapidement dans le chapitre suivant, lesquels couvrent de nombreux thèmes politiques et historiques, ainsi que des contraintes d'ordre moral en passant, par exemple, par l'interdiction qui pèse sur la psychanalyse. Cependant, malgré les tentatives d'identifier la responsabilité de telle ou telle institution ou de suivre l'évolution de certains tabous, cette partie offre plutôt une rapide esquisse de la question, série d'exemples et d'observations ponctuelles plutôt qu'une véritable étude analytique.

7 Une fois le cadre général, les acteurs, les modalités et les sujets d'intervention de la censure brejnévienne présentés, l'auteur fait le choix de proposer deux études de cas : d'abord, en comparant la version de Sandro de Čégem (Sandro iz Čegema) de Fazil' Iskander, publiée dans Novyj mir en 1973 avec celle parue en russe six ans plus tard chez l'éditeur américain Ardis, puis en étudiant l'histoire de l'almanach littéraire Metropol' qui, paru en 12 exemplaires en 1979, a provoqué un grand scandale et a conduit à des répressions à l'endroit de ses auteurs. Ces deux cas, analysés de façon fine et bien documentée, y compris grâce aux entretiens inédits avec les protagonistes, permettent à Maria Zalambani d'étudier au plus près l'action de la censure et, dans le second cas, de questionner le rôle de l'Union des écrivains en tant qu'organe de censure, de 
politique culturelle et de répression. Oscillations entre ligne " dure » et ligne « douce » de la politique idéologique soviétique des années 1970, conflits entre les institutions impliquées dans l'histoire de Metropol' ou encore impasses avouées par l'auteur quand elle souligne l'impossibilité d'identifier avec certitude l'origine de tous les écarts entre la version « soviétique » et la version « américaine » de Sandro de Čegem (Fazil' Iskander a probablement retravaillé lui-même son texte avant de le soumettre à l'action de l'éditeur-censeur de Novyj mir) - tout ceci rend compte de la complexité des phénomènes étudiés dans l'ouvrage et de la richesse de ces deux chapitres, sans doute les plus réussis. Notons cependant qu'ils ne sont pas exempts de ce qu'on pourrait considérer comme une lacune commune à la plupart des ouvrages consacrés à l'histoire de la censure littéraire soviétique. Maria Zalambani traite exclusivement de Moscou et évoque pour l'essentiel les cas les plus célèbres, tels que Solženitsyn ou Šolohov. Ce choix, sans doute assumé, laisse hors du champ de vision le travail routinier, banal, omniprésent, réalisé dans les maisons d'éditions de province ou à l'égard des écrivains qui, contrairement à Šolohov, ne saisissaient pas directement Brežnev pour s'enquérir du verdict de la censure...

Cette étude de la censure et de la politique littéraire brejnévienne se termine par un rapide aperçu de la disparition (elle, guère si rapide !) de la censure soviétique pendant la perestroïka. Il rend compte des tentatives de sauver les institutions de la censure, notamment le Glavlit, même sur fond de perte de légitimité et de désagrégation du système idéologique et politique soviétique.

9 Cette volonté de brosser un large tableau, articulée avec des tentatives systématiques de présenter un aperçu historique de chaque institution ou politique analysée (qui peuvent sans doute être utiles pour les lecteurs non-spécialistes dans le domaine russe) comportent naturellement des aspects de superficialité et des raccourcis parfois regrettables. On peut ainsi se demander s'il est vraiment pertinent de remonter à Pierre le Grand (p.71) pour raconter l'histoire de la police politique soviétique et surtout pour décrire son rôle dans l'exercice de la censure sous Brežnev (d'autant plus quand ceci est fait à partir d'un ouvrage de C. Andrew et O. Gordievskij publié en 1990). Remarquons à ce propos un usage quelque peu inégal de la littérature: si le texte et surtout la bibliographie montrent une très bonne connaissance des ouvrages récents, surtout russophones, consacrés aux sujets centraux de cette étude, les références semblent bien plus limitées et souvent datées quand il s'agit de domaines plus larges ou connexes, touchant à l'histoire politique et sociale de l'URSS. Quoi qu'il en soit, il s'agit d'un ouvrage fort appréciable, à l'écriture vivante, dont l'intérêt réside à la fois dans la volonté de synthèse, les analyses fines de cas concrets et la tentative de construire un cadre théorique pour étudier des questions souvent traitées ailleurs de façon descriptive.

\section{NOTES}

1. Nous nous limiterons à évoquer ici deux auteurs essentiels, qui ont publié de nombreuses monographies, articles et recueils d'archives sur le sujet : Arlen Bljum et Tat'jana Gorjaeva. 